\title{
De la Vallée Poussin's Extension of Poisson's Integral.
}

\author{
By George A. Gibson, M.A., LL.D.
}

The integrals of $\$ 5,6$, and 7 of the following paper were first established by C. de la Vallée Poussin in a memoir Sur quelques applications de l'intégrale de Poisson (Ann. de la Soc. sc. de Bruxelles, vol. 17, 1892-3). An analogous integral to that of $\$ 5$ was discovered by $A$. Hurwitz, who seems not to have been aware of de la Vallée Poussin's memoir, and will be found under the title Sur quelques applications des series de Fourier in the Annales de l'Ecole normale, vol. 19, 1902. In view of the value of these integrals for the theory of the Fourier series, the discussion now given, which follows different lines from those of previous proofs, may be of some interest. The discussion turns chiefly on the Second Theorem of Mean Value which is quite as applicable to Poisson's as to Dirichlet's Integral.

§1. Let $f(x)$ be given from $x=-\pi$ to $x=\pi$, and let it be defined for other values of $x$ by the equation $f(x)=f(x \pm 2 n \pi)$ where $n$ is a positive integer such that $-\pi<x \pm 2 n \pi \leqq \pi$. Suppose $f(x)$ to be either finite and integrable or else, when not finite, to be absolutely integrable, the number of discontinuities in the latter case being limited.

The integral, in which $0<r<1$,

$$
\phi(r, x)=\frac{1}{2 \pi} \int_{-\pi}^{\pi} \frac{f(u)\left(1-r^{2}\right) d u}{1-2 r \cos (u-x)+r^{2}}
$$

is a continous function of $x$ for any given $r$ less than 1; we wish to discuss some theorems involving the limit of $\phi(r, x)$ for $r$ converging to unity.

Since $f(u)$ is periodic we may, if convenient, write

$$
\phi(r, x)=\frac{1}{2 \pi} \int_{-\pi}^{\pi} \frac{f(u+x)\left(1-r^{2}\right) d u}{1-2 r \cos u+r^{2}}
$$

For brevity we shall sometimes put

$$
\left(1-r^{2}\right) /\left(1-2 r \cos (u-x)+r^{2}\right)=g(r, u-x) .
$$


\$2. Iet $h$ be any arbitrarily small but fixed positive number and consider the integrals

$$
\begin{gathered}
\mathrm{U}(r, x)=\frac{1}{2 \pi} \int_{x+h}^{\pi} f(u) g(r, u-x) d u, \mathrm{~V}(r, x)=\frac{1}{2 \pi} \int_{-\pi}^{x-h} f(u) g(r, u-x) d u, \\
\mathrm{~W}(r)=\frac{1}{2 \pi} \int_{-\pi+h}^{\pi-h} f(u) \frac{1-r^{2}}{1+2 r \cos u+r^{2}} d u .
\end{gathered}
$$

In the integrals $\mathrm{U}(r, x) \mathrm{V}(r, x) x$ may have any value in the range $(-\pi+h, \pi-h)$ and the sum $\mathrm{U}+\mathrm{V}$ gives for such $x$ the value of the integral in equation (1) except for the contribution arising from the range from $u=x-h$ to $u=x+h$ which constitutes the neighbourhood of $x$. The neighbourhood of the points $x= \pm \pi$ is to be considered as made up of the two parts from $-\pi$ to $-\pi+h$ and from $\pi-h$ to $\pi$; the integral $\mathrm{W}(r)$ gives the value of the integral in equation (1) for $x= \pm \pi$ except for the contribution arising from the neighbourhood of these points.

We show that as $r$ tends to unity $\mathrm{W}(r)$ tends to zero and $\mathrm{U}(r, x)$ and $\mathrm{V}(r, x)$ tend uniformly to zero.

Take $\mathrm{U}(r, x)$, which by putting $u+x$ for $u$ takes the form

$$
\mathrm{U}(r, x)=\frac{1}{2 \pi} \int_{h}^{\pi-x} f(u+x) \frac{1-r^{2}}{1-2 r \cos u+r^{2}} d u \text {. }
$$

The function $\left(1-r^{2}\right) /\left(1-2 r \cos u+r^{2}\right)$ or $g(r, u)$ is positive and decreasing from $u=0$ to $u=\pi$ but positive and increasing from $u=\pi$ to $u=2 \pi$; the second theorem of mean value can therefore be used. If $0 \leqq x \leqq \pi-h$ the function $g(r, u)$ is positive and decreasing within the range of integration and only one application of the theorem is needed; if $-\pi+h \leqq x<0$, then $x$ is negative and $\pi-x$ is greater than $\pi$ so that two applications are needed.

Suppose $x$ to be negative; then

$$
\begin{aligned}
& \mathrm{U}(r, x) \\
& =\frac{1}{2 \pi} \int_{h}^{\pi} f(u+x) \frac{1-r^{2}}{1-2 r \cos u+r^{2}} d u+\frac{1}{2 \pi} \int_{\pi}^{\pi-x} f(u+x) \frac{1-r^{2}}{1-2 r \cos u+r^{2}} d u \\
& =a+\beta, \operatorname{say}, \\
& a=\frac{1-r^{2}}{2 \pi\left(1-2 r \cos h+r^{2}\right)} \int_{h}^{\xi} f(u+x) d u, h \leqq \xi \leqq \pi
\end{aligned}
$$

since $g(r, u)$ is in this case a positive decreasing function ;

$$
\beta=\frac{1-r^{2}}{2 \pi\left(1+2 r \cos x+r^{2}\right)} \int_{\xi^{\prime}}^{\pi-x} f(u+x) d u, \pi \leqq \xi^{\prime} \leqq \pi-x
$$

since $g(r, u)$ is now a positive increasing function. 
The integrals

$$
\int_{h}^{\xi} f(u-x) d u, \int_{\xi^{\prime}}^{\pi-x} f(u+x) d x
$$

are each numerically less than the integral

$$
\int_{-\pi}^{\pi}|f(u)| d u
$$

which is finite and independent of $x$.

The factor $\left(1-r^{2}\right) / 2 \pi\left(1-2 r \cos h+r^{2}\right)$ converges to zero as $r$ converges to unity since $h$ is not zero. (It is easy to see that if $h$ is not fixed but tends to zero as $r$ tends to unity the limit is still zero provided that the limit of $(1-r) / h^{2}$ is zero when $r$ tends to unity.) The factor $\left(1-r^{2}\right) / 2 \pi\left(1+2 r \cos x+r^{2}\right)$ is not greater than $\left(1-r^{2}\right) / 2 \pi\left(1+2 r \cos h+r^{2}\right)$ and therefore tends to zero as $r$ tends to unity whatever value $x$ may have within the range to which it is confined.

Thus $\mathrm{U}(r, x)$ tends uniformly to zero as $r$ converges to unity. Similar considerations hold for the integrals $\mathrm{V}(r, x)$ and $\mathrm{W}(r)$.

We have therefore the result that if $h$ is any fixed positive number, no matter how small if it be different from zero, and $x$ any number such that $-\pi+h \leqq x \leqq \pi-h$, it is always possible to take $r$ so near to unity that the sum

$$
|\mathrm{U}(r, x)+\mathrm{V}(r, x)|
$$

will become and, as $r$ is taken closer to unity, will remain less than any given arbitrarily small positive number.

\$3. Let us suppose for the present that $f(x)$ is finite and integrable, the case of infinite discontinuities being considered later. Make a division of the range from $-\pi$ to $\pi$ and consider those inter $; a l s, e_{s}$ say, in which the oscillation of $f(x)$ exceeds $\sigma$, where $\sigma$ is any arbitrarily small positive number. Since $f(x)$ is integrable it must be possible to choose the intervals $e_{\text {a }}$ so that $\Sigma e_{\text {, }}$ will be less than any given arbitrarily small positive number $\epsilon$; let $\sigma, \epsilon$ be given and the intervals $e_{s}$ determined.

Next enclose each interval $e$, in an interval $e_{s}^{\prime}$ so that $e_{s}^{\prime}$ overlaps $e_{\varepsilon}$ at both ends and let $\Sigma e_{s}^{\prime}$ be less than $2 \epsilon$; denote by $A^{\prime}$ what is left of the range $(-\pi, \pi)$ when the intervals $e_{s}^{\prime}$ have been removed. The extremities $-\pi$ and $\pi$ will not usually belong to $A^{\prime}$ but must be assigned to $e_{s}^{\prime}$. 
We now choose the number $h$ of $\$ 2$; we take $h$ to be any fixed number less than the least distance from an extremity of $e_{s}$ to the corresponding extremity of the $e_{a}^{\prime}$ which overlaps it. When $h$ is so chosen we shall have

$$
|f(x+u)-f(x)| \leqq \sigma \text { if } 0 \leqq|u| \leqq h
$$

for every $x$ in $\mathrm{A}^{\prime}$.

Now let $x$ be any number in $A^{\prime}$.

$$
\phi(r, x)=\mathrm{U}(r, x)+\mathrm{V}(r, x)+\frac{1}{2 \pi} \int_{x-h}^{x+h} f(u) g(r, u-x) d u .
$$

The last term in this equation may be written

$$
\begin{aligned}
& \frac{1}{2 \pi} \int_{x}^{x+h} f(u) g(r, u-x) d u+\frac{1}{2 \pi} \int_{x-h}^{x} f(u) g(r, u-x) d u \\
= & \mathrm{I}_{1}+\mathrm{I}_{2} \text {, say. } \\
\mathrm{I}_{1}= & \frac{1}{2 \pi} \int_{0}^{h} f(x+u) g(r, u) d u \\
= & \frac{f(x)}{2 \pi} \int_{0}^{h} g(r, u) d u+\frac{1}{2 \pi} \int_{0}^{h}\{f(x+u)-f(x)\} g(r, u) d u .
\end{aligned}
$$

By taking $r$ close enough to unity the first term may be made to differ as little as we please from $\frac{1}{2} f(x)$. Next

$$
\frac{1}{2 \pi}\left|\int_{0}^{h}\{f(x+u)-f(x)\} g(r, u) d u\right| \leqq \frac{\sigma}{2 \pi} \int_{0}^{h} g(r, u) d u
$$

and therefore

$$
\leqq \frac{1}{2} \sigma
$$

whatever value less than unity is assigned to $r$. Thus we can choose $\rho^{\prime}$ so that when $\rho^{\prime}<r<1$

$$
\left|I_{1}-\frac{1}{2} f(x)\right|<\frac{1}{2} \sigma+\frac{1}{2} \sigma, \text { that is, }<\sigma ;
$$

and, in exactly the same way, it follows that when $\rho^{\prime \prime}<r<1$

$$
\left|I_{2}-\frac{1}{2} f(x)\right|<\sigma \text {. }
$$

By $\$ 2, \rho^{\prime \prime \prime}$ can be chosen so that when $\rho^{\prime \prime \prime}<r<1$ the sum $|\mathrm{U}(r, x)+\mathrm{V}(r, x)|$ will be as small as we please, say less than $\sigma$.

Thus we have shown that if $x$ is any number in $A^{\prime}$ we can choose a number $\rho$ such that when $\rho<r<1$

$$
|\phi(r, x)-f(x)|<3 \sigma
$$

in other words, when $x$ is any number in $\mathrm{A}^{\prime}, \phi(r, x)$ converges uniformly to $f(x)$. 
If $x$ is not in $\mathrm{A}^{\prime}$ let $\mathrm{M}$ be an upper limit to the values of $f(x)$; then

$$
|\phi(r, x)|<\frac{\mathbf{M}}{2 \pi} \int_{-\pi}^{\pi} g(r, u-x) d u \leqq \mathbf{M}
$$

so that $|\phi(r, x)|$ is finite for every $r$.

It is important to observe that, whether $f(x)$ has infinite discontinuities or not, $\left|I_{1}+I_{2}\right|$ is finite for every $r$ less than unity when $x$ is not in the neighbourhood of a point of infinite discontinuity. For if $\mathrm{M}$ is an upper limit to the values of $|f(u)|$ from $u=x-h$ to $u=x+h$

$$
\left|\mathrm{I}_{1}+\mathrm{I}_{2}\right|<\frac{\mathbf{M}}{2 \pi} \int_{x-h}^{x+h} g(r, u-x) d u \leqq \mathbf{M} .
$$

Hence as $r$ tends to unity $|\phi(r, x)|$ remains finite, because $|\mathrm{U}(r, x)+\mathrm{V}(r, x)|$ may be made as small as we please.

\$4. Let $\mathrm{F}(x)$ satisfy the conditions stated for $f(x)$ in $\$ 1$; when $F(x)$ is finite and integrable from $x=-\pi$ to $x=\pi$ the function $\psi(r, x)$, defined by the equation

$$
\psi(r, x)=\frac{1}{2 \pi} \int_{-\pi}^{\pi} \mathrm{F}(v) g(r, v-x) d v
$$

converges uniformly to $F(x)$, when $r$ tends to unity, for every number $x$ in the range $\mathrm{A}^{\prime \prime}$, corresponding to $\mathrm{A}^{\prime}$, and in the other parts of the range is finite, less than or equal to the upper limit of $|F(x)|$.

\$5. When $f(x)$ and $\mathrm{F}(x)$ are finite and integrable

$$
\mathrm{L}_{r=1} \int_{a}^{b} \phi(r, x) \psi(r, x) d x=\int_{a}^{b} f(x) \mathrm{F}(x) d x
$$

where $-\pi \leqq a<b \leqq \pi$.

Denote by $\mathrm{B}^{\prime}$ what is left of the range $(a, b)$ after removal of the intervals $e_{t}^{\prime}$, determined now for both functions $f(x)$ and $F(x)$. If $x$ is any number in $\mathrm{B}^{\prime}$ the product $\phi(r, x) \psi(r, x)$ converges uniformly to the product $f(x) \mathrm{F}(x)$ and therefore

$$
\underset{r=1}{\mathrm{~L}} \int_{\mathrm{B}^{\prime}} \phi(r, x) \psi(r, x) d x=\int_{\mathrm{B}^{\prime}} f(x) \mathrm{F}(x) d x
$$

the integration extending over the range $\mathrm{B}^{\prime}$, that is, from $a$ to $b$ but excluding the invervals $e_{a}^{\prime}$. 
Suppose $|f(x)|$ and $|\mathrm{F}(x)|$ each less than $M$ for every $x$ not in $\mathrm{B}^{\prime}$; then the product $|\phi(r, x) \psi(r, x)|$ is less than $\mathrm{M}^{2}$ for every such $x$. Hence

$$
\left|\int_{\mathbb{E}^{\prime}} \phi(r, x) \psi(r, x) d x\right|<\mathbf{M}^{2} \Sigma_{e^{\prime}}^{\prime} ;\left|\int_{\mathbf{E}^{\prime}} f(x) \mathrm{F}(x) d x\right|<\mathbf{M}^{2} \Sigma_{e_{\iota}}
$$

the integration extending over all the intervals $e_{a}^{\prime}$. But $\Sigma e_{a}^{\prime}$ can be made as small as we please, so that equation (2) becomes

$$
\underset{r=1}{\mathrm{~L}} \int_{a}^{b} \phi(r, x) \psi(r, x) d x=\int_{a}^{b} f(x) \mathbf{F}(x) d x .
$$

$\$ 6$. Suppose now that $f(x)$ and $\mathrm{F}(x)$ have infinite discontinuities ; it will be sufficient to suppose $f(x)$ infinite for $x=c$ and $F(x)$ infinite for $x=c^{\prime}$. The functions are assumed to be absolutely integrable. When $c$ and $c^{\prime}$ are unequal the product $f(x) \mathrm{F}(x)$ will also be absolutely integrable; but when $c$ and $c^{\prime}$ are equal it does not necessarily follow that the product is absolutely integrable so that when $c=c^{\prime}$ the express hypothesis is made that the product is absolutely integrable. The theorem of $\$ 5$ holds in both cases, as will now be seen.

Equation (2) of $\$ 5$ is still true provided $B^{\prime}$ denote the range $(a, b)$ with the neighbourhoods of $c$ and $c^{\prime}$ removed. To establish the theorem therefore we have merely to show that the contribution to the integrals of equation (1) $\$ 5$ from values of $x$ in the neighbourhood of $c$ and $c^{\prime}$ can be made as small as we please by diminishing the extent of the neighbourhood.

First suppose $c$ and $c^{\prime}$ unequal. The integrals

$$
\left|\int_{c-\eta}^{c+\eta^{\prime}} f(x) \mathrm{F}(x) d x\right|,\left|\int_{c^{\prime}-\eta}^{c^{\prime}+\eta} f(x) \mathrm{F}(x) d x\right|
$$

may by choice of $\eta$ and $\eta^{\prime}$ be made arbitrarily small, because the product $f(x) \mathrm{F}(x)$ is absolutely integrable.

When $x$ is in the neighbourhood of $c,|\psi(r, x)|$ is less than a finite number $M$, and

$$
\begin{aligned}
\phi(r, x) & =\frac{1}{2 \pi} \int_{x-h}^{x+h} f(u) g(r, u-x) d u+\gamma \\
& =\mathrm{I}+\gamma, \text { say, }
\end{aligned}
$$


where $\gamma$ tends uniformly to zero as $r$ tends to unity. Now

$$
\begin{aligned}
\left|\int_{c-\eta}^{c+\eta^{\prime}} \mathrm{I} \psi(r, x) d x\right| & \leqq \frac{\mathrm{M}}{2 \pi}\left|\int_{c-\eta}^{c+\eta^{\prime}} d x \int_{-h}^{h} f(x+u) g(r, u) d u\right| \\
& \leqq \frac{\mathrm{M}}{2 \pi}\left|\int_{-h}^{h} g(r, u) d u \int_{c-\eta}^{c+\eta^{\prime}} f(x+u) d x\right| \\
& <\mathrm{M} \epsilon
\end{aligned}
$$

by proper choice of $\eta$ and $\eta^{\prime}$, because $f(x+u)$ is absolutely integrable.

Next

$$
\left|\int_{c-\eta}^{c+\eta^{\prime}} \gamma \psi(r, x) d x\right|<\mathbf{M}\left|\gamma_{1}\right|\left(\eta+\eta^{\prime}\right)
$$

where $\left|\gamma_{1}\right|$ is an upper limit to $|\gamma|$.

Thus

$$
\begin{aligned}
\left|\int_{c-\eta}^{c+\eta^{\prime}} \phi(r, x) \psi(r, x) d x\right| & =\left|\int_{c-\eta}^{c+\eta^{\prime}}(\mathrm{I}+\gamma) \psi(r, x) d x\right| \\
& <\mathrm{M}_{\epsilon}+\mathrm{M}\left|\gamma_{1}\right|\left(\eta+\eta^{\prime}\right)
\end{aligned}
$$

and can therefore be made arbitrarily small.

A similar investigation is applicable when $x$ is in the neighbourhood of $c^{\prime}$, so that the theorem of $\$ 5$ still stands.

Next, suppose $c$ and $c^{\prime}$ to be equal. The integral

$$
\left|\int_{c-\eta}^{c+\eta^{\prime}} f(x) \mathrm{F}(x) d x\right|
$$

may be made arbitrarily small by choice of $\eta$ and $\eta^{\prime}$.

We also need the result that if $-h \leqq u \leqq h,-h \leqq v \leqq h$ the integral

$$
\left|\int_{c-\eta}^{c+\eta^{\prime}} f(x+u) \mathrm{F}(x+v) d x\right|
$$

may be made arbitrarily small (less than $\epsilon$ ) by choice of $\eta, \eta^{\prime}$. Whether $u$ and $v$ are equal or not the product $f(x+u) \mathrm{F}(x+v)$ is ubsolutely integrable and the result is evident.

If we write

$$
\psi(r, x)=\frac{1}{2 \pi} \int_{x-h}^{x+h} \mathrm{~F}(v) g(r, v-x)+\delta=\mathrm{J}+\delta
$$

and put $\mathrm{I}+\gamma$ for $\phi(r, x)$ as before, we may obviously disregard $\gamma$ and $\delta$, as a process precisely similar to that just used will show. 
We have therefore to examine the integral of $I \times J$.

$$
\begin{aligned}
\int_{c-\eta}^{c+\eta^{\prime}} \mathrm{IJJ} d x & =\frac{1}{4 \pi^{2}} \int_{c-\eta}^{c+\eta^{\prime}} d x \int_{-h}^{h} f(x+u) g(r, u) d u \int_{-h}^{h} \mathrm{~F}(x+v) g(r, v) d v \\
& =\frac{1}{4 \pi^{2}} \int_{-h}^{h} g(r, u) d u \int_{-h}^{h} g(r, v) d v \int_{c-\eta}^{c+\eta^{\prime}} f(x+u) \mathrm{F}(x+v) d x .
\end{aligned}
$$

Thus

$$
\left|\int_{c-\eta}^{c+\eta} \mathrm{IJ} d x\right|<\frac{\epsilon}{4 \pi^{2}} \int_{-h}^{h} g(r, u) d u \int_{-h}^{h} g(r, v) d v
$$

and therefore less than $\epsilon$.

The theorem of $\$ 5$ is thus true under the conditions stated. Of course if there were more than one infinity, the process would be repeated for each, so that the theorem holds when the number of points of infinite discontinuity is finite.

\$7. If $\chi(x)$ is finite and integrable, the more general equation holds, namely,

$$
\underset{r=1}{\mathrm{~L}} \int_{a}^{b} \phi(r, x) \psi(r, x) \chi(x) d x=\int_{a}^{b} f(x) \mathrm{F}(x), \chi(x) d x
$$

as is very readily seen after what has been done. 